\title{
Research on the Action Design of Team Artistic Swimming
}

\author{
Li Li ${ }^{1}$, Xu Xin ${ }^{1}, \mathrm{Mu} \mathrm{Wei}^{2}$, Dong $\mathrm{Hui}^{3}$, Pang Mei ${ }^{4}$ \\ ${ }^{1}$ PE Teacher's Office, Guangdong Food and Drug Vocational College, Guangzhou, China \\ ${ }^{2}$ Sports Department, Tianjin University of Science \& Technology, Tianjin, China \\ ${ }^{3}$ Physical Education Department, Hebei University DPER, Baoding, China \\ ${ }^{4}$ Martial Arts and Dance Academy, Shenyang Sport University, Shenyang, China
}

\section{Email address:}

wozaifeitianwu@126.com (Li Li), 154002353@qq.com (Xu Xin), 53265040@qq.com (Mu Wei), 343857012@qq.com (Dong Hui), 466483223@qq.com (Pang Mei)

${ }^{*}$ Corresponding author

\section{To cite this article:}

Li Li, Xu Xin, Mu Wei, Dong Hui, Pang Mei. Research on the Action Design of Team Artistic Swimming. International Journal of Sports Science and Physical Education. Vol. 5, No. 1, 2020, pp. 5-9. doi: 10.11648/j.ijsspe.20200501.12

Received: March 9, 2020; Accepted: April 15, 2020; Published: May 14, 2020

\begin{abstract}
By the methods of interview and literature consultation, this paper analyzes the action arrangement and design of Artistic Swimming. The research shows that although the existence of water allows the appearance of only half of the swimmer's body in the team Artistic Swimming, the reasonable action design and arrangement can still make the complete set of movements novel, diversified and artistic. In the specific arrangement and design, the pictographic action can add to the resemblance of the action to realistic items both in "shape" and "manner"; story action can show a certain theme and situation; difficult action is the concrete embodiment of athletes' ability and strength; original action can make the complete set of action unique and more attractive; coordinating action reflects the athletes' mutual assistance and cooperation. Through the cooperation between swimmers, the styles and types of Artistic Swimming are enriched, and so are the action arrangements.
\end{abstract}

Keywords: Artistic Swimming, Team Event, Action Design, Research

\section{Introduction}

Action language expresses the inner impulse and thought of life through body posture, human expression (including facial expression) and the time, space and gravity changes of human body in motion. As for the arrangement and design of Artistic Swimming, because of the existence of water, the movements of Artistic Swimming are seen through the half body exposed above the water, which can only be displayed either by the upper limb movements while the legs are paddling underneath, or by the leg movements while arms are rowing underneath. It appears that the half body movement is the whole body language of Artistic Swimming. But in fact, the body part hidden in the water are the "birthplace" of strength, movement and even emotion. On the one hand, the rowing support of the leg movement, the paddling support of the arm movement, and the display of the strength of the action all depend on the participation of the body part hidden in the water; on the other hand, the body part kept underwater for most of the time is also responsible for expressing the body language and the action emotion. The separate display of upper limb and leg movements does not affect the artistic appreciation of Artistic Swimming. Through drawing on various artistic elements, combined with reasonable design and arrangement, Artistic Swimming is as innovative, diversified and artistic as other sports and art events.

Analyzing the different designs of various Artistic Swimming movements and exploring their types and characteristics can not only enrich the theoretical research of Artistic Swimming, but also provide practical reference for the arrangement and action design of Artistic Swimming in the future.

\section{Research Methods}

\subsection{Interview}

In order to clarify the research ideas and determine the 
research patterns, in-depth interviews were conducted with experts in Artistic Swimming (international A-level referees), scholars (university professors), dance teachers, music producers, experts in art design and aesthetic research, and leaders in charge of Artistic Swimming in the swimming management center of the General Administration of Sport of China.

\subsection{Literature Consultation}

(1) According to the research content of this paper, relevant literatures from 1980 to 2016 were searched from CNKI, ProQuest Dissertation Full-text Retrieval Platform (http://pqdt.calis.edu.cn) and Springer online journal (http://link.springer.com) to obtain relevant paper and electronic documents.

(2) Collecting the competition rules of Artistic Swimming over the years to understand the development process of the event comprehensively, so as to "know the history, understand the current situation and predict the future".

(3) Referring to related works in aesthetics, visual arts, music, dance and sports training to provide theoretical support.

\section{Research Results and Analysis}

\subsection{Pictographic Action}

In the design of Artistic Swimming, the theme and music should be interpreted, so the performance of the action must be displayed with symbolic meaning. Artistic Swimming is much like dance, poetry, painting and many other art forms. It is an epitome of the scene in life and stresses both the "manner" and "shape". Through carefully designed movements, athletes transform the visualized things and scenes around us into abstract pictographic movements with symbolic meanings so as to interpret the music and the theme. Because of the limitation of the swimming pool, if the athletes want to show more content in the limited space, they need to arrange and design the pictographic action that fits the theme, make full use of the limited action language to achieve the maximum performance, and use the symbolic action to interpret the music and theme they want to convey. Gestalt Psychology points out that for a figure or object, when it is similar to another figure or object in one aspect, people's visual sense and perception tends to form a whole of various parts according to past experience. According to this, if the choreographed and designed actions in team Artistic Swimming want to achieve the purpose of making the audience feel the "image" and "manner" after watching the show, it is necessary to make the choreographed and designed actions have some similarity in image with the things to be displayed, so that people can quickly catch the "similar" information, thus producing resonance.

In the team free competition of London Olympic Games in 2012, the Chinese team presented the theme Butterfly. After entering the water, the girls used the instep contact to straighten up their legs after a difficult lift. Then they used the same instep contact posture to open their legs into a shape similar to a crossbar, and outlined the wings of the butterfly with such leg actions. After that, they jumped up from the "wings" they presented and extended their arms upward, just like a butterfly breaking through the shackles and breaking out of the cocoon. The Spanish team performed the theme Ocean. At the beginning of the water action, they had a set of $4 \times 4$ cooperative action. The four girls, hand in hand, leaped from the water sideway and then into the water, which was like the scene of a whale rushing out of the horizontal plane and then into the water. In addition, in the upper limb movement, whether it was the water paddling with both arms on top of the water or the leg kicking on the back, the Spanish team added a lot of elbow bending and wrist pressing postures to show the fish's double gills; in order to highlight the theme of "ocean", they also made corresponding changes in the layout and design of their leg movements. Their legs in the handstand posture were no longer tightened and straightened, but changed to an overlapping position. They also changed the stretched instep into an open hook. The changes in the leg formation no longer display the regular ballet performance, but the dance like a fish tail in the water. Here, the movements displayed by body language are consistent with the artistic conception conveyed by the music theme. In the arrangement and design, symbolic imagery (for example, two members of the Chinese team broke out of the cocoon with "butterfly wings" erected by the legs of other members) and virtual imagery (for example, the fish tail and gill posture when the Spanish team displayed the "ocean" theme) are often used to convey the symbolic meanings of the movements. This kind of action design that imitates something by using arm and leg is actually to use body language as a "form", and find out its definite similarity with something in this form, and then design and symbolize it through action performance. Finally, what we see through visual senses in actual life is reproduced through this symbolic action, so that people can understand its symbolic meaning.

\subsection{Story Action}

In the arrangement and design of team Artistic Swimming, the complete set of actions are not pictographic actions that imitate some things, but story actions that depict a situation, which are often used in the action design. If the pictographic action is to express the theme through the "shape" and "manner" of various actions, then the story action is to narrate the "story" and highlight the theme by using the speed and range of the body movement, the emotion and expression of the face, and the conventional way of expression that corresponds to the public psychology.

At the end of the 19th century, French educator and dance theorist Francis del Sartre created the "expression system", that is, to make a systematic and scientific analysis of body movements and expressions. This theory provides important enlightenment for the understanding of the expressive function of different parts of the body, and for the analysis of human personality through the dynamic characteristics of the body. The research of modern dance theorist and educator 
Rudolph von Laban provides a more systematic theory. He observed: "the amazing human structure and the magical activities it may perform are the greatest miracles that exist in the world. Every human movement, every change of center of gravity and every simple gesture of any part of human body show some characteristics of our life. Every action is brought about by the activation of the nerve. The conscious or subconscious action impulse is formed due to either the real-time sensory impression, or the complex chain of the sensory impression that has been experienced in the past". At the end of his dance works, Laban came to the conclusion that it is possible to explain the whole life of human beings with the inner activity reflected by body feeling. Therefore, it is possible to show different artistic conception and theme through different actions in the arrangement and design of team free Artistic Swimming. In terms of dance creation, we can use the human body as a medium to reflect our cognition of things. Artistic Swimming, as a sports as well as an art event, is not an absolute competitive sports event that pursues "higher, faster and stronger". Instead, it is committed to reflecting the competitive sports ability, and at the same time, uses multiple art forms to express the music theme and emotion with abstract body language, and finally expresses "beauty". In the leg action, the fast, powerful and changeable movement is to display a situation of vitality, joy or surprise; the stretching and extending of the main body and upper limb movement are to reflect the emotional scene embodied with melancholy, sadness and lingering; and the greater range movement involving all the body is an emotional control, which can show the situation of anger and thrill. Through the story actions of team Artistic Swimming, those music themes that can not be translated by human language are interpreted by body actions to showcase people's inner thoughts, so that the audience can understand the themes with the help of the actions, and then imagine the artistic conception. In such a way, the unfolding of the story and the performance of the action are perfectly combined to arouse an aesthetic enjoyment.

In the London Olympic Games, the Russian team free Lost World was created from a film of the same name. In the complete set of actions, they showed the fear of a team of explorers when they encountered dinosaurs in an undeveloped land with tight and fast leg movements, and the anxiety of explorers with bending and stretching movements of double legs. Their heads down, shoulders down and arms half raised showed the situation of people's worry, fear and terror. They also conveyed explorers' efforts and insistence with continuous rotating movements and expressed their struggle to find the road to modern civilization with large swing of their upper limbs. The now fast motion and then large swing of body movements, coupled with the constant change of facial expressions, enabled the audience to feel all kinds of situations of the film and appreciate the wonderful and unique action design of the Russian team.

\subsection{Difficult Action}

As a competitive and artistic sports event, Artistic
Swimming must bear both difficulty and artistry. Actions supported only by difficulty will have less artistry. Similarly, purely artistic design without difficulty is uncompetitive and unattractive. Difficult actions in team Artistic Swimming are the embodiment of the athletes' strong ability and a way to show the flexible and ingenious action design. In team Artistic Swimming, difficult actions that can show the ability of athletes mainly include: leg movements with compound changes in spine position (such as rotation movements with the ears, hips and ankles on the same vertical line. Because of the curvature of the spine, these movements change into a position that requires more strength and stability to fit the curved spine); leg movements with changes in the center of gravity ( such as the greater range and multi-angle leg movements); leg movements with the largest range of flexibility (such as rushing splits, front and back jete); continuous movements with arms on the water surface and complicated changing angles; team cooperative actions with various postures; blind postures where the athletes can not see each other; lifting actions with overlapping, throwing and jumping, and different dimensions of rotations. Difficult actions are accomplished through continuous changes of body postures, the shifts of the center of gravity, the transformation of space and time and the imbalance and balance presented by the body in the movement, and the flexible and ingenious cooperation between the athletes. Surely, difficult actions can not be achieved without the competitive techniques of the athletes themselves, but they are also related to the flexible and ingenious action design. No matter what kind of difficulty, action design has to be presented by actions to show the artistic conception of the theme. The actions of the athletes must be "clean and neat" as well as "beautiful and excellent". Because as a competitive sport, Artistic Swimming requires certain difficulty and intensity of the athlete's actions; and as a sports and art event, Artistic Swimming requires the athletes to be able to attract and surprise the audience with every move of their actions.

In the 2013 World Swimming Championship, the Russian team performed the same theme as in the 2012 London Olympics, but this did not hinder their excellent performance. In the middle stage of the whole process (approaching the third line after entering the water), they had a series of difficult and complex leg movements: they first completed four pairs of double teammates actions of $2 \times 8$ beats in a back-to-back blind posture. Meanwhile, they shifted between the high and low positions (up and down) only by the contact with their teammate's toes. The high position movement needs more strength and support, while the low position movement needs to be completed in control and stability. Such "high" and "low", "up" and "down" movements not only reflect the difficulty and strength of the action, but also showcase the scene of struggle between people and the outside world in the theme film Lost World. After the team cooperative action, what came next was a series of greater range leg movements combined with curved spine movements, which required different parts of the body, movement direction and rhythm to change at the same time. 
In addition, along with the "contraction and extension" of the legs, the spine also needed to bend and stretch correspondingly to complete various actions. This process witnessed the rotation of the spine and constant change of the body center. Contraction (bending of the spine) brought tension and energy accumulation in the body, while extension (stretching of the spine) enabled the body to extend both internally and externally. After a strong, great range and tense abdomen retraction, it was more difficult to stretch the spine. Such action design allowed the audience to feel the difficulty of the movements, and at the same time, it also proved the athletes' extraordinary techniques. In addition, it transmitted to the audience a strong sense of internal conflict and external impact, which can be an excellent way to express a theme with great conflicts or dramatic changes.

\subsection{Original Action}

If we see Artistic Swimming only as a kind of sport, then it is composed of pure human body movements; if we consider Artistic Swimming as bearing a certain degree of artistry, it should be a kind of sports and art form that is not expressed by the language medium, but uses the body language to convey and express the main theme. It is a sport with high artistic appreciation value among all the competitive sports. In Artistic Swimming, especially in the team event, the original action is the highlight. It is the unique design and wonderful original actions that separate Artistic Swimming from other competitive sports, and make the team event more attractive and appreciative.

The design of original action needs the designer's careful observation of daily life and continuous accumulation of materials to make sure the Artistic Swimming action is unique and adheres to the characteristics of "water". It also needs rich imagination, which requires the designers to recombine and rearrange the established actions and sometimes make additional processing in an effort to create actions that have never existed based on the action image kept in their memory, or the experience of previous designs. In this process, the transformation and combination is the embodiment of creativity, and the additional processing also relies on the previous experience of design. The design of highlight action is neither about "dancing" to the music, nor about the design of a coherent action, but about ambitious design and innovation. Therefore, it needs the athletes' excellent techniques and ability, as well as certain innovative thinking techniques.

In the 16th World Artistic Swimming Championship in 2015, the Russian team free was ingenious, especially their several lifts, which left a deep impression on the audience. The trend of Artistic Swimming today is that the lift is accomplished by the "top" players who are lifted above the water head first or feet first, and then make somersault moves in different dimensions at the highest point, so as to show the adventure and difficulty of the action. But this is not the case for the lifts designed by the Russian team. Apart from the first lift after they entered the water which was followed by vertical somersaults in the high point, the following several lifts were all different. In the second big lift, they adopted a posture in groups of four that enabled them to move on the plane and rotate. In the third lift, they changed from an inverted position to an upright position with rotation, bringing a fresh action image to people. Such a unique way of design aims to seek "difference", to impress and attract people with unusual and unconventional performance. From the perspective of innovative thinking, being original is to create a unique set of actions by means of diffusion, reverse, and transformation thinking. In the process of the design, we should aim to develop different actions and should not follow the trend blindly. We ought to discard similarities and seek differences, so as to create a new, different and unprecedented action design. In addition, in the middle stage of this set of actions, the Russian team had a series of leg movements that changed from straight line to double teammate cooperation, and then to two oblique rows. In this leg combination, they adopted the same movements, with different leg lifts and opposite movement directions. At the same time, their leg movements are great range movements of straight leg swings. With graceful music, they moved and changed formation fast. Their bodies stretched freely with strength and energy surging. Their legs extended as far as they could, creating an aesthetic scene featured by slender figure, dynamic stretch, vigorous strength, rich changes, fluency and harmony, allowing people to feel the smoothness and elegance. This set of actions can be called the "magic" combination of leg movements. The aim of the design is something "new", which is embodied by novelty in innovative thinking. The new action design can catch people's eyes and bring people a fresh and curious feeling. Novelty focuses on seeking something "new" and reflecting "new ideas". It should be different from the conventions, unheard of and unprecedented, so as to achieve the purpose of innovation and surprise.

\subsection{Coordinating Action}

Team cooperation is one of the characteristics of team Artistic Swimming. In the solo event, all the actions are performed by one person, without contact with any partners or teammates. Double event is confined within the cooperation between two people. Only in the team event can the cooperation among the athletes be all-round and multidimensional. The cooperation can be conducted in groups of two, three, four, five and eight. Compared with the solo event and the double event, the team event can produce different artistic effects with the cooperation and mutual assistance among the athletes. The contact between athletes' bodies is a kind of "dialogue" rather than "control"; it is a cooperative relationship rather than mutual exclusion; it is a kind of trust rather than doubt; it is a new form of action generated by force rather than a "fight" that offsets each other.

Susan Langer, a famous philosopher and one of the representatives of semiotic aesthetics, pointed out in her book Problems of Art that: "when we watch dance, we see the interaction of forces, but this force is not the kind of gravity 
that weight has, nor the kind of thrust used to push books down, but the kind of sheer force that seems to promote the dance itself. In a double dance, two people seem to be attracted by a kind of force and tightly connected into one; in a group dance, all people seem to be attracted and inspired by the same central force or energy".

In the coordinating action of Artistic Swimming, the athletes are connected as one through mutual assistance and cooperation. They are connected by their arms, legs or make a move of bilateral symmetry, so as to create a new action different from that of a single person. In this case, the coordinating action not only presents the physical force through human body, but also is a new way to interpret the music theme for the athletes. The mutual cooperation between athletes reflects not only the dialectical unity of various forces and actions, but also the ultimate beauty of their actions. The correspondence and cooperation of body postures can make a connection between the external postures and internal relations of actions, thus forming action symbols which are not only expressive but also full of internal meaning. Therefore, this is a good way to express the theme and create aesthetic atmosphere, where the audience's infinite imagination is aroused by the tangible cooperation image, thus triggering a dynamic movement space, as well as a psychological space with aesthetic orientation.

In the 16th World Artistic Swimming Championship, the Ukrainian team adopted more coordinating and team actions. After the entering water lift, they completed a series of leg movements in groups of two in a crossed formation, followed by a set of symmetrical movements of eight people. In addition, in their whole set of movements, they sometimes formed in groups of two, sometimes in groups of three and sometimes eight of them acted together as a whole. The flexible and ingenious design make their movements not abrupt, but more changeable. We can say that coordinating action is more expressive and artistic than the individual action. It enriches the styles and types of Artistic Swimming action through the cooperation between people, making Artistic Swimming action more diversified.

\section{Conclusion}

In the design of team Artistic Swimming, because of the existence of water, the movements of Artistic Swimming are seen through only the half body exposed above the water. Nevertheless, understanding different action patterns and using rich movement changes can also make the design of actions more diversified and attractive. Exploring the action design of team Artistic Swimming can help enrich the theoretical research of Artistic Swimming and provide practical reference for the arrangement and design of Artistic Swimming in the future.

\section{References}

[1] Kurt Koffka, Principles of Gestalt Psychology, Beijing: Peking University Press, October, 2016, pp: 98.

[2] Qingyi Liu, Body Language Tutorial for Modern Dance, Beijing: China Renmin University Press, May, 2011, pp: 31.

[3] Bingting Luo, Design of Free Collective Item of Synchronized Swimming in Rio Olympic Games, 33rd ed., vol. 2. Journal of sports Adult Education, 2017, pp: 83-85.

[4] Yuan Wang, Aiping Chen, Research on 3D Space Utilization of the Free Optional Motion Exercise in Synchronized Swimming, 34th ed., vol. 6. Journal of Guangzhou Sport University, 2014, pp: $88-91,98$

[5] Tong $\mathrm{Xu}$, (2013) School of Social Sports, Aesthetic Interpretation of Reasons for the Artistic Trend of Contemporary Sports. Journal of Shenyang Sport University, 2013, 4.

[6] http://wenku.baidu.com/view/c5e2a3fdf705cc1755270930.ht $\mathrm{ml}$ [EB/OL]: 2011.05.14

[7] http://zhidao.baidu.com/link?url=ODYbciUANjm91ml2DW9i 9122xthm_qpKTcjoLVgJB1i_6K2jnjQ4yYti2hDqTvLTafcM0 5I-jFdcuGu95trDuK [EB/OL] : 2019.09.11

[8] http://blog.sina.com.cn/s/blog_513be6d60102wbwv.html [EB/OL]: 2015.12.12.

[9] Dong yue Wang, (2011) Research on the Evaluation Model of Model of Excellent Synchronized Swimming Athletes' Figure in China. The International Symposium on Applied Economics, Business and Development.

[10] Senigaglia Valeria; (2012) The role of synchronized swimming as affiliative and anti-predatory behavior in long-finned pilot whales. Behavioural processes, 9: 8-14.

[11] Lundy Bronwen, (2011) Nutrition for synchronized swimming: a review. International journal of sport nutrition and exercise metabolism, 10: 43-45.

[12] Ponciano Kátia; (2018) Physiological responses during the practice of synchronized swimming: a systematic review. Clinical physiology and functional imaging, 3: 163-175.

[13] Zenic Natasa; (2016) Comparative analysis of substance use in ballet, dance sport, and synchronized swimming: results of a longitudinal study. Medical problems of performing artists, 6 : 75-81.

[14] Filgueiras Alberto; Hall Craig R; (2017) Psychometric properties of the Brazilian-adapted version of Sport Imagery Questionnaire. Psicologia, reflexao e critica: revista semestral do Departamento de Psicologia da UFRGS, 10: 22.

[15] Ye Liu, Li Yu, Dongmei Luo, (2010) Analysis of the rockets technique in China synchronized swimming team with three-dimensional kinematic approach. 16th Asian games science congress. 